\title{
Comparison of three magnetic nanoparticle tracers for sentinel lymph node biopsy in an in vivo porcine model
}

This article was published in the following Dove Press journal:

International Journal of Nanomedicine

II February 2015

Number of times this article has been viewed

\author{
Joost J Pouw ${ }^{1, *}$ \\ Muneer Ahmed ${ }^{2}, *$ \\ Bauke Anninga ${ }^{2}$ \\ Kimberley Schuurman' \\ Sarah E Pinder ${ }^{2}$ \\ Mieke Van Hemelrijck ${ }^{3}$ \\ Quentin A Pankhurst ${ }^{4,5}$ \\ Michael Douek ${ }^{2}$ \\ Bennie ten Haken'
}

'MIRA Institute for Biomedical Technology and Technical Medicine, University of Twente, Enschede, the Netherlands; 'Research Oncology, Division of Cancer Studies, King's College London, Guy's Hospital, London, UK; ${ }^{3}$ Cancer Epidemiology Group, Division of Cancer Studies, King's College London, London, UK; ${ }^{4}$ Healthcare Biomagnetics Laboratory, University College London, London, UK; ${ }^{5}$ Institute of Biomedical Engineering, University College London, London, UK

*These authors contributed equally to this work

Correspondence: Bennie ten Haken MIRA Institute for Biomedical Technology and Technical Medicine, University of Twente, PO Box 217, 7500AE Enschede, the Netherlands

Tel +3। 0534892158

Email b.tenhaken@utwente.nl

Michael Douek

Research Oncology, Division of Cancer Studies, King's College London, Guy's

Hospital, Great Maze Pond, London SEI

9RT, UK

Tel +44207l886380

Email michael.douek@kcl.ac.uk
Introduction: Breast cancer staging with sentinel lymph node biopsy relies on the use of radioisotopes, which limits the availability of the procedure worldwide. The use of a magnetic nanoparticle tracer and a handheld magnetometer provides a radiation-free alternative, which was recently evaluated in two clinical trials. The hydrodynamic particle size of the used magnetic tracer differs substantially from the radioisotope tracer and could therefore benefit from optimization. The aim of this study was to assess the performance of three different-sized magnetic nanoparticle tracers for sentinel lymph node biopsy within an in vivo porcine model.

Materials and methods: Sentinel lymph node biopsy was performed within a validated porcine model using three magnetic nanoparticle tracers, approved for use in humans (ferumoxytol, with hydrodynamic diameter $d_{\mathrm{H}}=32 \mathrm{~nm}$; Sienna $+{ }^{\circledR}, d_{\mathrm{H}}=59 \mathrm{~nm}$; and ferumoxide, $d_{\mathrm{H}}=111 \mathrm{~nm}$ ), and a handheld magnetometer. Magnetometer counts (transcutaneous and ex vivo), iron quantification (vibrating sample magnetometry), and histopathological assessments were performed on all ex vivo nodes.

Results: Transcutaneous "hotspots" were present in 12/12 cases within 30 minutes of injection for the $59 \mathrm{~nm}$ tracer, compared to $7 / 12$ for the $32 \mathrm{~nm}$ tracer and 8/12 for the $111 \mathrm{~nm}$ tracer, at the same time point. Ex vivo magnetometer counts were significantly greater for the $59 \mathrm{~nm}$ tracer than for the other tracers. Significantly more nodes per basin were excised for the $32 \mathrm{~nm}$ tracer compared to other tracers, indicating poor retention of the $32 \mathrm{~nm}$ tracer. Using the $59 \mathrm{~nm}$ tracer resulted in a significantly higher iron accumulation compared to the $32 \mathrm{~nm}$ tracer.

Conclusion: The $59 \mathrm{~nm}$ tracer demonstrated rapid lymphatic uptake, retention in the first nodes reached, and accumulation in high concentration, making it the most suitable tracer for intraoperative sentinel lymph node localization.

Keywords: superparamagnetic iron oxide, breast cancer, magnetic tracer, ferumoxytol, ferumoxide, Sienna $+^{\circledR}$

\section{Introduction}

Breast cancer is the most common cancer among women, with approximately 1.67 million new patients diagnosed annually worldwide and accounting for $25 \%$ of all cancer cases. ${ }^{1}$ Breast cancer predominantly spreads via the lymphatic system to locoregional lymph nodes, meaning that the status of these lymph nodes is important for staging of the disease and determining prognosis..$^{2-4}$

The sentinel lymph nodes (SLNs) are defined as the first draining nodes from a primary tumor and, therefore, are most likely to be the first site of lymphatic metastasis. Sentinel lymph node biopsy (SLNB) with the "combined technique" of radioisotope and blue dye is the standard method for determining axillary staging in early-stage breast cancer patients with a clinically and radiologically negative axilla., ${ }^{3,5-9}$ 
The combined technique is performed by injecting a radioactive tracer ${ }^{99 \mathrm{~m}} \mathrm{Tc}$ nanocolloid) and blue dye interstitially, either peritumorally or periareolarly. The tracers are distributed through the lymphatic system, to the draining SLNs. The tracers are then detected within the SLNs by the surgeon using a handheld scintillation counter (gamma probe) and/or visually. The gamma probe is first used to transcutaneously localize the SLNs and determine the optimal incision site. Postincision both the blue dye and gamma probe guide the surgeon to the SLNs. After identification, the SLNs are removed and sent for histopathological examination. With an identification rate of $96 \%$ and a false-negative rate of $5 \%-10 \%$, it is an effective procedure with low morbidity. ${ }^{3}$

Although the currently used technique performs well, it has drawbacks. The use of radioisotopes is subject to stringent regulations on training of staff and handling and disposal of radioactive material. Furthermore, the 6 hour half-life of ${ }^{99 \mathrm{~m}} \mathrm{Tc}$ limits theater scheduling. Most importantly, many hospitals in the world do not have access to radioisotopes. Consequently, only approximately $60 \%$ of the patients in the western world and negligible numbers in the rest of the world have access to SLNB. ${ }^{10}$ This has led to the search for other techniques, not reliant on the use of radioisotopes. ${ }^{11}$

Recently the use of a magnetic tracer and a handheld magnetometer was evaluated against the "combined technique" as a radiation-free alternative in two separate clinical trials. ${ }^{12,13}$ A superparamagnetic iron oxide (SPIO) tracer was administered interstitially in the breast, followed by identification of the SLNs with a handheld magnetometer. The magnetic technique was found to be noninferior to the "combined technique" with identification rates of $94.4 \%-$ 98.0\%. ${ }^{12,13}$ However, there was a discordance in SLNs identified between the magnetic and combined techniques that ranged between $2.0 \%{ }^{13}$ and $6.9 \%,{ }^{12}$ - potentially leading to false-negative staging with the magnetic technique. A smaller study by Shiozawa et $\mathrm{al}^{14}$ used a similar SPIO tracer and different magnetometer, comparing SPIO and blue dye only - no radioisotope was used. They found an SLN identification rate of $90 \%$ for the SPIO in combination with blue dye, with a discordance rate of $16.7 \%$ and demonstrated the viability of the technique for performing SLNB in the absence of radioisotopes.

Physical and chemical properties including shape, coating material, and particle size influence the distribution of nanoparticles in vivo. ${ }^{15,1699 \mathrm{~m}} \mathrm{Tc}$ nanocolloid has a mean particle size of approximately $8 \mathrm{~nm},{ }^{17,18}$ while the particle size of the magnetic tracer used for the magnetic technique (Sienna ${ }^{\circledR}$, Endomagnetics Ltd, UK) is much larger with a mean size of approximately $60 \mathrm{~nm}$. Because particle size is the most important property influencing lymphatic uptake and lymph node retention, ${ }^{19}$ the identification of different SLNs by the two techniques could possibly arise due to differences in particle size of the radioisotope and magnetic tracer. In order to optimize the clinical application of the magnetic technique for SLNB, we evaluated the performance of three different-sized SPIO-based formulations - licensed for human use - within an in vivo porcine model.

\section{Materials and methods}

\section{Tracers/dynamic light scattering}

Three SPIO-based licensed formulations with a broad range in particle size were used as magnetic tracers in this study. Feraheme $^{\circledR} /$ Rienso $^{\circledR}$ (Takeda, Japan) consists of ferumoxytol, $30 \mathrm{mg} \mathrm{Fe} / \mathrm{mL}$ and is an Food and Drug Administration-approved drug used for the intravenous treatment of iron deficiency anemia in adult patients with chronic kidney disease. The particles have a magnetic core of maghemite $\left(\gamma-\mathrm{Fe}_{2} \mathrm{O}_{3}\right)$ surrounded by a polyglucose sorbitol carboxymethylether coating. Sienna $+{ }^{\circledR}$ contains $27 \mathrm{mg} \mathrm{Fe} / \mathrm{mL}$ and is a CE-marked magnetic tracer intended to mark and locate SLNs in cancer patients. The magnetic core consists of magnetite and maghemite $\left(\mathrm{Fe}_{3} \mathrm{O}_{4} / \gamma-\mathrm{Fe}_{2} \mathrm{O}_{3}\right)$, with a carboxydextran coating. Finally, Endorem ${ }^{\circledR} /$ Feride $^{\circledR}$ (Guerbet, France) consisting of ferumoxide, $11.2 \mathrm{mg} \mathrm{Fe} / \mathrm{mL}$ is licensed as a magnetic resonance imaging (MRI) contrast agent for liver imaging, with a magnetite core coated with dextran.

All magnetic tracers were diluted to the same iron concentration of $11.2 \mathrm{mg} \mathrm{Fe} / \mathrm{mL}$ to facilitate comparison of performance. Sterile water for injection and a pipette were used to prepare the dilutions. The hydrodynamic particle size of all tracers was determined by dynamic light scattering using a Zetasizer Nano ZS (Malvern Instruments, UK).

\section{Animals and surgery}

Ethical permission for animal experimentation was granted by the Research Institute against Digestive Cancer (IRCAD) Ethics Review Board (Strasbourg, France) under reference number 38.2012.01.047. A previously developed and validated porcine model that closely resembles human lymphatic drainage was used to evaluate the performance of the different magnetic tracers for SLNB. ${ }^{20}$ At the IRCAD Institute (Strasbourg, France), after induction of anesthesia, mini-pigs were injected with one of the three different magnetic tracers subcutaneously bilaterally into the areolas of the third inguinal mammary glands. A total of $0.5 \mathrm{~mL}(5.6 \mathrm{mg} \mathrm{Fe})$ of each diluted tracer was injected on each side, in six mini-pigs, for each tracer (18 mini-pigs in total; 36 injections). 
Transcutaneous magnetometer measurements using a handheld magnetometer (SentiMAG ${ }^{\circledR}$, Endomagnetics Ltd, UK) were undertaken in the inguinal region, where the draining lymph nodes are located. Measurements were performed at 5 minutes, 10 minutes, 15 minutes, 30 minutes, 45 minutes, 60 minutes, and 240 minutes after administration of the tracer. The sites at which uptake of SPIO was successfully detected (hotspots) were marked using a permanent marker.

Bilateral SLNB of the inguinal region was undertaken 4 hours after injection. The incision was made at the site of the "magnetic hotspot". If no "hotspot" was located, a blind incision was made in the inguinal region. Postincision, the handheld magnetometer was used to identify the SLNs in vivo. After identification, lymph nodes demonstrating SPIO uptake were removed, and the ex vivo magnetometer count was recorded. Lymph nodes with a magnetometer count higher than $10 \%$ of the "hottest node" were defined as SLNs, as was used in the SentiMAG Multicentre Trial, ${ }^{12}$ and in accordance with standard clinical practice. ${ }^{21}$

After completion of the SLNB procedure, a groin node clearance was performed. Ex vivo magnetometer measurements were performed on the groin node clearances to determine if there were any further SLNs that were missed initially. All resected lymph nodes were fixed in formalin and sent to the University of Twente (Enschede, The Netherlands) for iron quantification and subsequently to King's College London (London, UK) for histopathological evaluation.

\section{Quantification of iron content (vibrating sample magnetometry)}

The quantification of magnetic tracer in the excised and formalin-fixed sentinel lymph nodes was performed using vibrating sample magnetometry (VSM) on a Physical Properties Measuring system (Quantum Design Inc., San Diego, CA, USA). An applied magnetic field of $4.0 \mathrm{~T}$ was used to bring the magnetic iron oxide nanoparticles to saturation. The amount of magnetic tracer in the lymph nodes was determined by comparing the obtained amplitude of the saturation magnetization to known calibration samples of each of the tracers. The iron content was reported as the mass of iron $(\mathrm{Fe})$ in the node, present in the form of maghemite or magnetite. VSM allows quantification with $0.5 \mu \mathrm{g} \mathrm{Fe}$ accuracy and is nondestructive, which allows subsequent histopathological analysis of the same samples. ${ }^{22}$

\section{Histopathology}

The nodes were then transferred intact to King's College London (London, UK) where they were embedded in wax, thinly sliced, and stained with hematoxylin and eosin and with Perl's Prussian blue for iron. Iron staining within each node was graded using a previously validated 5 -point grading scale $(0=$ none, $1=$ minimal, $2=$ mild, $3=$ moderate, and $4=$ marked $),{ }^{20}$ by an experienced pathologist and a second observer (SP, BA, or MA).

\section{Statistical analysis}

We conducted a two-sided test ( alpha $=0.05$ ) expecting a difference of $50 \mu \mathrm{g}$ (standard deviation $[\mathrm{SD}]=30$ ) in iron content readings between each magnetic tracer. When performing a total of 12 procedures (six mini-pigs) for each magnetic tracer, these 36 procedures provided us with a power of $82 \%$ to detect this difference. The relationship between continuous variables was calculated using Pearson's correlation coefficient, associations between categorical data using the $\chi^{2}$ test, and associations between categorical and continuous variables using analyses of variance. All statistical analyses were performed using Statistical Analysis Systems (SAS) release 9.3 (SAS Institute, Cary, NC).

\section{Results \\ Dynamic light scattering}

The Z-averaged hydrodynamic diameter of the magnetic tracers ferumoxytol, Sienna $+{ }^{\circledR}$, and ferumoxide was determined to be $32 \mathrm{~nm}, 59 \mathrm{~nm}$, and $111 \mathrm{~nm}$, respectively. When the size distributions were evaluated graphically (Figure 1), significant intratracer heterogeneity in particle size was observed. This is reflected by a polydispersity index of $0.179,0.181$, and 0.266 for the $32 \mathrm{~nm}$ tracer, $59 \mathrm{~nm}$ tracer, and $111 \mathrm{~nm}$ tracer, respectively.

\section{Surgery}

A total of 36 SLNB procedures were performed in 18 minipigs, and SLN identification was successful in all cases. In vivo magnetic hotspots from the draining inguinal lymph nodes were successfully identified transcutaneously prior to surgical excision using the handheld magnetometer in all cases (12/12) for the $32 \mathrm{~nm}$ tracer and the $59 \mathrm{~nm}$ tracer and in all but one case (11/12) for the $111 \mathrm{~nm}$ tracer (240 minutes after injection). The number of procedures in which a transcutaneous hotspot was successfully identified increased with time after injection for all three tracers (Figure 2). There were $7 / 12$ and $8 / 12$ successfully identified magnetic hotspots after 30 minutes using the $32 \mathrm{~nm}$ tracer and $111 \mathrm{~nm}$ tracer, respectively, compared to the $59 \mathrm{~nm}$ tracer with magnetic hotspots in all cases (12/12). 


\section{Particle size distribution of the tracers}

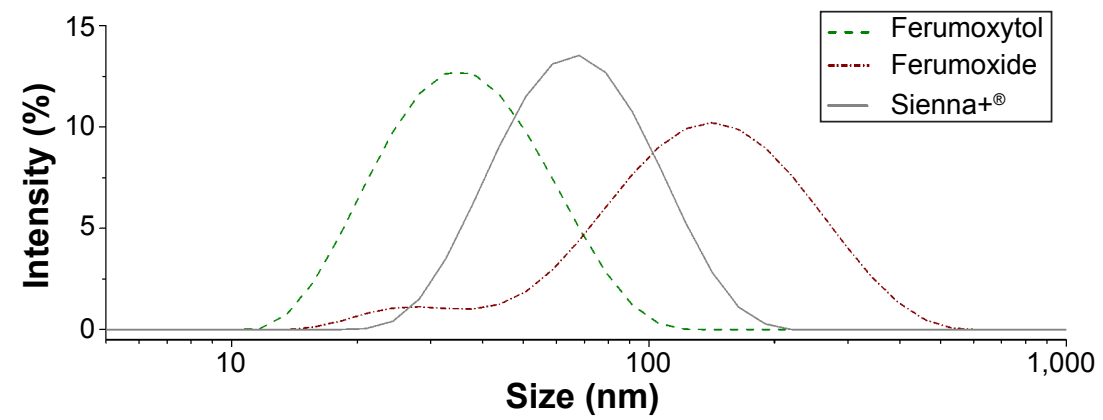

Figure I Particle size distribution of the three magnetic tracers by dynamic light scattering. Note: The particle size (logarithmic scale) is shown against the intensity.

A total of 77 SLNs were identified with the handheld magnetometer during surgery and subsequently excised. Thirty-five SLNs were identified using the $32 \mathrm{~nm}$ tracer (mean 2.9, SD 1.2), 20 SLNs with the $111 \mathrm{~nm}$ tracer (mean 1.7, SD 0.9), and 22 SLNs (mean 1.8, SD 0.8) with the 59 nm tracer (Figure 3A). A statistically significant difference between the tracer used, and the number of SLNs identified was observed overall ( $P=0.0099)$ (Figure 3A). More nodes per basin were excised with the $32 \mathrm{~nm}$ tracer compared to the $111 \mathrm{~nm}$ tracer $(P<0.001)$ and the $59 \mathrm{~nm}$ tracer $(P=0.03)$.

The distribution of magnetometer counts demonstrated a statistically significant difference among the three tracers overall ( $P<0.0001)$ (Figure 3B). Multicomparisons demonstrated that ex vivo magnetometer counts were significantly higher with the $59 \mathrm{~nm}$ tracer compared to the other two tracers $(P<0.05)$.

\section{Quantification of iron content (VSM)}

The iron content of the ex vivo SLNs was recorded using VSM (Figure 3C). Multiple comparisons between the tracers demonstrated a significant higher iron content for the $59 \mathrm{~nm}$ tracer compared to the $32 \mathrm{~nm}$ tracer $(P<0.05)$ only. The mean iron content of the SLNs was determined to be 106 (SD 70) $\mu \mathrm{g}, 179$ (SD 159) $\mu \mathrm{g}$, and 265 (SD 206) $\mu \mathrm{g}$ for the $32 \mathrm{~nm}$ tracer, $111 \mathrm{~nm}$ tracer, and $59 \mathrm{~nm}$ tracer, respectively. A linear relationship between the iron content of the ex vivo SLNs and the handheld magnetometer counts was observed for all tracers (Figure 4) - $32 \mathrm{~nm}$ tracer ( $r=0.93$; $P<0.001), 111 \mathrm{~nm}$ tracer $(r=0.95 ; P<0.001)$, and $59 \mathrm{~nm}$ tracer $(r=0.93 ; P<0.001)$. Figure 4 demonstrates a difference in the sensitivity of the magnetometer for the different tracers. The magnetometer count is 2.8 times higher for the $59 \mathrm{~nm}$ tracer compared to the same amount of iron for the $111 \mathrm{~nm}$ tracer and 1.3 times higher for the $32 \mathrm{~nm}$ tracer compared to the $111 \mathrm{~nm}$ tracer.

\section{Histopathology}

The iron was distributed predominantly in the subcapsular space peripherally within the cortex, subcapsular space, and sinuses (Figure 5). The iron deposition from the $111 \mathrm{~nm}$

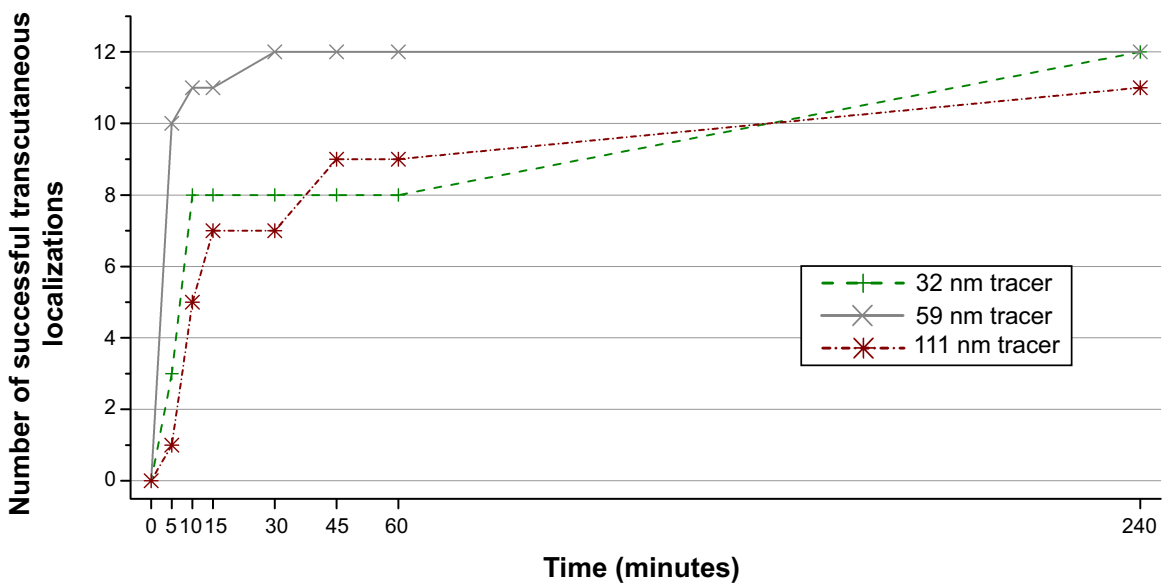

Figure 2 Relationship between the time after injection of the magnetic tracer and successful identification of transcutaneous hotspot. 

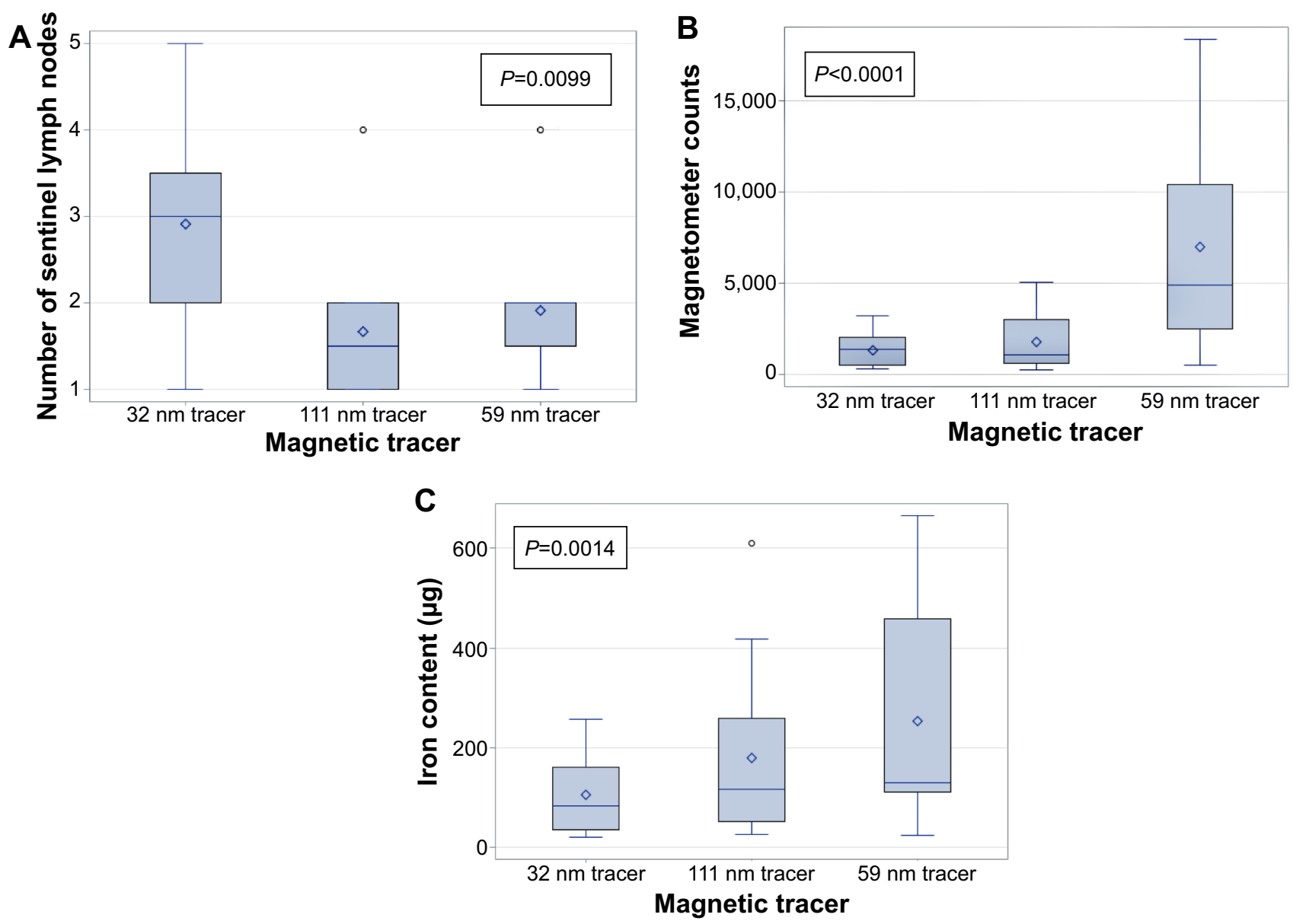

Figure 3 Boxplots of the relationship between the magnetic tracer and: (A) the number of excised sentinel lymph nodes during surgery; (B) the ex vivo magnetometer counts of the excised sentinel lymph nodes; $(\mathbf{C})$ the iron content measured of the excised sentinel lymph nodes by vibrating sample magnetometry.

Notes: The circle symbols represent outliers; the diamond symbols represent the mean value.

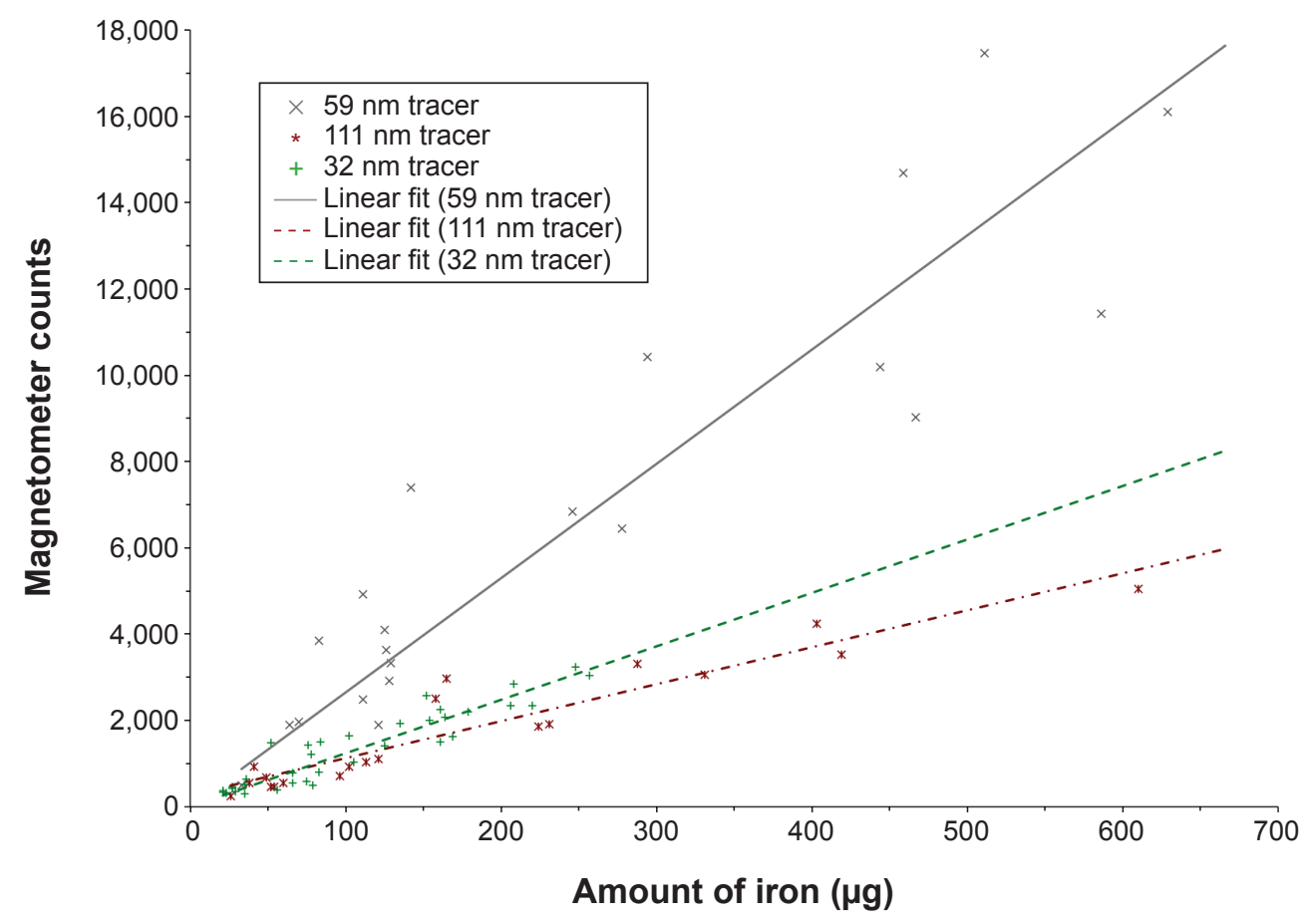

Figure 4 Correlation between the iron content as determined by vibrating sample magnetometry and the ex vivo magnetometer counts for the different magnetic tracers. 

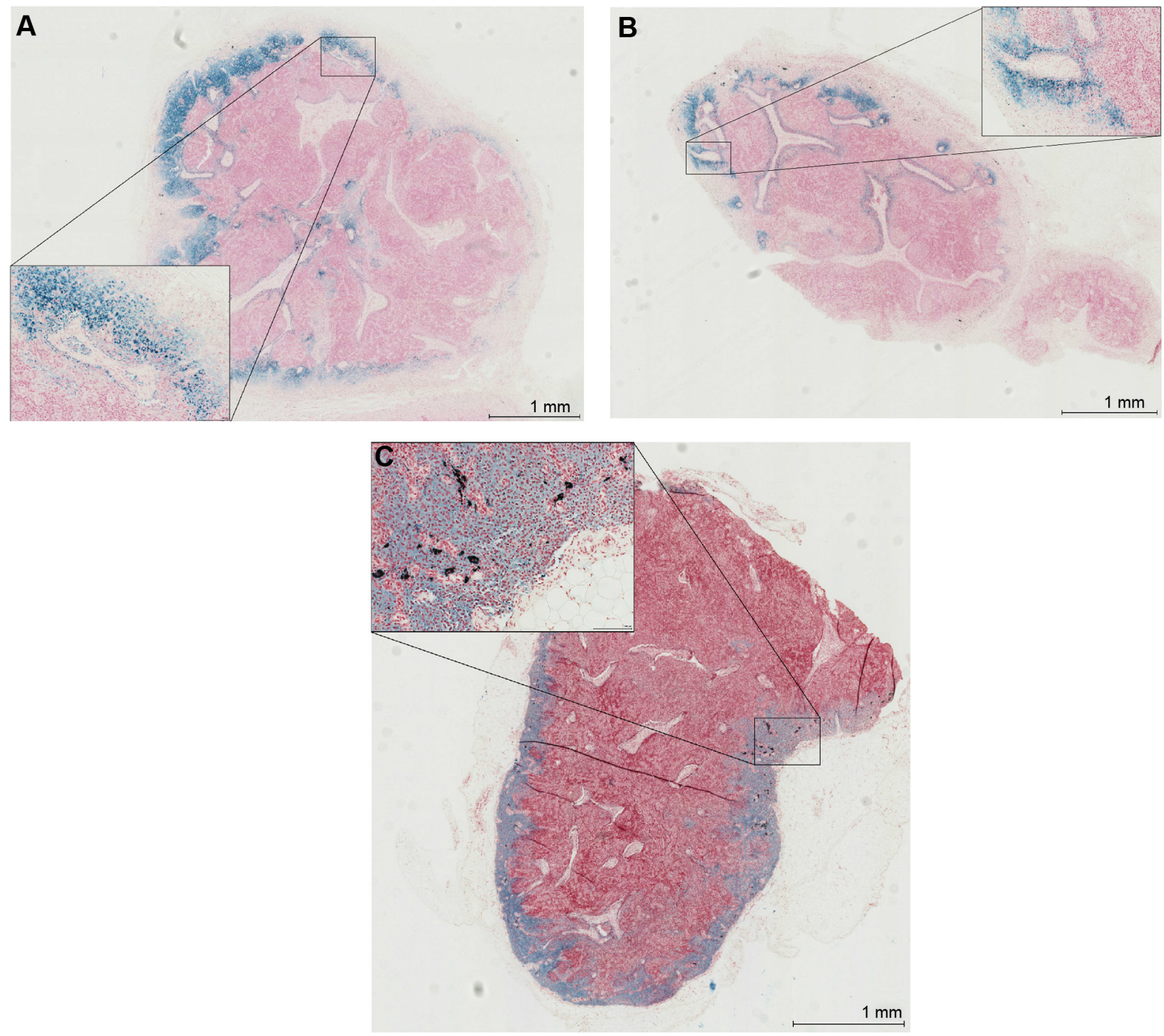

Figure 5 Iron distribution within nodes on histopathology using Perl's Prussian blue staining for iron and haematoxyline \& eosine.

Notes: Magnification $2 \times$, with inserts at $20 \times$ magnification. (A) Node containing the III-nm tracer; (B) node containing the $59 \mathrm{~nm}$ tracer; (C) node containing the $32 \mathrm{~nm}$ tracer.

tracer was mostly confined to macrophages within the nodes (Figure 5A), whereas the $59 \mathrm{~nm}$ tracer demonstrated more free iron granules (particularly peripherally) (Figure 5B). Only very sparse, small islands of iron within macrophages were visible for the $32 \mathrm{~nm}$ tracer (Figure 5C). There was a significant difference in the grade of the iron content of excised SLNs between the three different tracers overall $(P<0.0001)$ (Figure 6). The amount of iron deposition was significantly greater with the $111 \mathrm{~nm}$ tracer and $59 \mathrm{~nm}$ tracer $(P<0.05)$ compared to the $32 \mathrm{~nm}$ tracer.

\section{Discussion}

Rapid uptake of a magnetic tracer after interstitial injection is important to maximize transcutaneous hotspot detection and enable injection in the theater suite, facilitating theater scheduling. Transcutaneous identification of hotspots was possible in most procedures with the $59 \mathrm{~nm}$ tracer within 10 minutes of the injection and in all except one procedure (with the $111 \mathrm{~nm}$ tracer) within 240 minutes. Within 30 minutes of injection, all hotspots were already identifiable using the $59 \mathrm{~nm}$ tracer. The greater sensitivity of the magnetometer for the $59 \mathrm{~nm}$ tracer for a given quantity of iron compared to the other two tracers (Figure 4) facilitates hotspot detection.

Discoloration of the skin was present at the injection site using all tracers, but this was not quantified during this study. Our animal model did not allow to evaluate whether the skin discoloration would fade away over time or persist as a tattoo. Although superficial administration of an SPIO tracer 


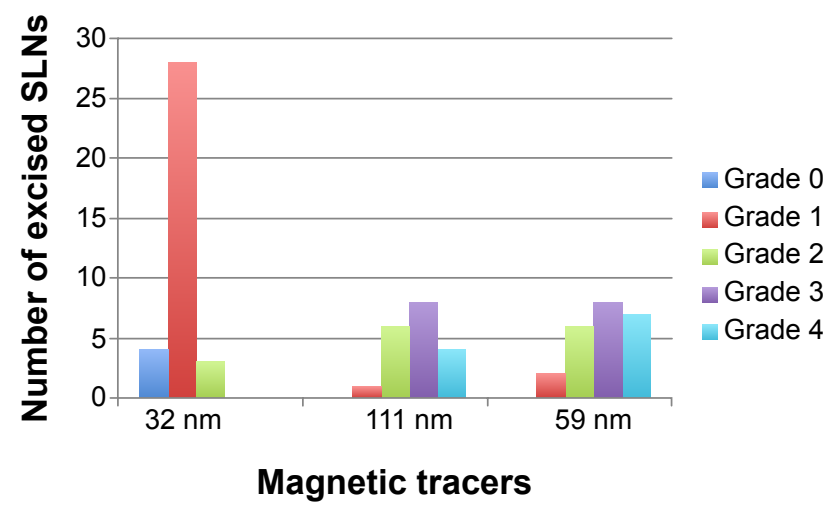

Figure 6 Number of excised sentinel lymph nodes (SLNs) per grade of iron distribution, for the three different magnetic tracers.

Note: Grade 0 = none, Grade I = minimal, Grade $2=$ mild, Grade 3 = moderate and Grade 4 = marked.

can result in skin tattooing, the current clinical studies using SPIO have also not reported adequate follow-up data to fully quantify this. ${ }^{12-14}$ However, any tattooing is anticipated to be temporary rather than permanent but may persist for several months - similar to that expected for blue dye. ${ }^{23}$

Any tracer used for SLNB must be retained by the SLNs and not pass on to higher echelon nodes in order to avoid excessive excision of normal nodes. Within this study, a statistically significantly greater number of nodes were excised using the $32 \mathrm{~nm}$ tracer compared to the other two tracers. This is a distinct disadvantage of the smaller particle-sized magnetic tracer. It suggests that rather than being retained within the SLN after entering through the afferent duct, it passes through the efferent system and onto higher echelon nodes. This is reflected by the small islands of sparsely distributed iron within the nodes after the administration of the $32 \mathrm{~nm}$ tracer on histopathology (Figure 5C). By applying the " $10 \%$ rule" and excising those nodes with only $10 \%$ of the ex vivo count of the hottest excised node using radioisotope, Martin et $\mathrm{al}^{24}$ demonstrated that a mean SLN harvest of 1.96 nodes was associated with a false-negative rate of $5.8 \%$. In the SentiMAG Multicentre Trial using the $59 \mathrm{~nm}$ tracer, 2.02 nodes were excised per procedure using the magnetic technique. Excising more than two SLNs during sentinel node biopsy is not proven to be beneficial except after primary systemic therapy ${ }^{25}$ Our data suggest that the small-sized 32 $\mathrm{nm}$ tracer would result in removal of an excessive number of normal nodes.

In addition to retention, the accumulation of magnetic tracer within the SLNs is essential for intraoperative identification. The $59 \mathrm{~nm}$ tracer demonstrated statistically significant higher ex vivo magnetometer counts compared to the other two tracers, but only a statistically significant greater iron content over the $32 \mathrm{~nm}$ tracer. This is explained by the higher sensitivity of the used magnetometer for the $59 \mathrm{~nm}$ tracer compared to the other two tracers. The higher sensitivity for the $59 \mathrm{~nm}$ tracer is due to differences in magnetic properties of the tracers, arising from differences in size and material of the magnetic cores. ${ }^{26}$

By injecting the magnetic tracer 24 hours before surgery, one may be able to improve the iron uptake by the SLNs and thereby improve the performance of a tracer. However, extending the period between injection and surgery could allow adverse SPIO migration to higher echelon nodes beyond the SLN. Therefore, this is likely only suitable for the larger $111 \mathrm{~nm}$ tracer - within this context. However, to optimize the time between injection and surgery for each tracer, formal assessment of individual tracers for SLNB when injected at different times before surgery is needed.

The variation in the accumulation of iron within nodes, between different tracers was demonstrated on histopathology. There was significantly less iron deposition in nodes from the $32 \mathrm{~nm}$ tracer compared to the other tracers, with only sparse, small islands of iron derived from the $32 \mathrm{~nm}$ tracer being observed in the nodes. There was no difference in the grading of nodes between the $59 \mathrm{~nm}$ tracer and the $111 \mathrm{~nm}$ tracer on histopathology. In both cases, the iron was distributed in the subcapsular space and sinuses of nodes, consistent with previous histopathological studies..$^{20,27}$ However, the $111 \mathrm{~nm}$ tracer was found to be deposited mainly within macrophages - demonstrating its ability to activate the mononuclear phagocytic system - compared to the $59 \mathrm{~nm}$ tracer, which although demonstrating phagocytosis within the node also displayed extensive free iron granules peripherally. These two processes of activating the mononuclear phagocytic system and free iron granule deposition facilitate iron accumulation and hence SLN identification using a handheld magnetometer.

Different studies have recently evaluated the influence of magnetic nanoparticle size on lymphatic uptake in rodent models. ${ }^{28-30}$ Tracers with particle sizes ranging from 4 to $1,000 \mathrm{~nm}$ were used in these studies. The purpose of these studies was to optimize tracer uptake in the SLNs to facilitate preoperative localization with MRI, rather than intraoperative detection. Mori et al ${ }^{30}$ used $50 \mathrm{~nm}, 100 \mathrm{~nm}, 200 \mathrm{~nm}$, and $1,000 \mathrm{~nm}$-sized particles and concluded that particles of $200 \mathrm{~nm}$ and larger are not suitable in view of lack of uptake within 24 hours. Iida et a ${ }^{28}$ compared the performance of $4 \mathrm{~nm}, 8 \mathrm{~nm}$, and $20 \mathrm{~nm}$ citrate-coated nanoparticles and concluded that the $20 \mathrm{~nm}$ particles were best retained in the SLNs and accumulated in the highest concentration. However, the $20 \mathrm{~nm}$ tracer was the largest used, and therefore, it is possible 
that uptake could be further optimized by increasing the particle size. Kjellman et $\mathrm{al}^{29}$ used monodisperse magnetic nanoparticles with a polyethylene glycol coating with sizes of $15 \mathrm{~nm}, 27 \mathrm{~nm}$, and $57 \mathrm{~nm}$. The $15 \mathrm{~nm}$ particles were observed to accumulate in the SLNs the fastest and in the highest concentrations. A $15 \mathrm{~nm}$ polyethylene glycol-coated tracer approved for use in humans is currently not available and was not used in our study. Therefore, the efficiency of the $15 \mathrm{~nm}$ tracer cannot be compared to our results.

During the course of the animal experimentation, there were no signs of adverse events developing within the animals as a consequence of the magnetic tracer administration. When magnetic tracers are used as MRI contrast agents, doses of 25-100 mg are typically administered. Animal studies have demonstrated no acute or subacute toxicity when 150 times this dose has been administered. ${ }^{31}$ Consequently, iron overload would not be possible within this model due to the small amounts of iron injected $(5.6 \mathrm{mg})$.

In this study we evaluated the performance in SLNB of three magnetic tracers approved for human use. However, only the $59 \mathrm{~nm}$ tracer is currently CE-marked for the purpose of SLNB in Europe. The $111 \mathrm{~nm}$ and $32 \mathrm{~nm}$ tracers are licensed as an MRI contrast agent and an intravenous treatment of iron deficiency anemia, respectively. Apart from the dilution of the tracers to standardize iron concentrations, no changes were made to the constituents to facilitate the translation of the results to clinical practice. The formulation of the tracers is very similar; all are aqueous suspensions of magnetic nanoparticles in water, with a $\mathrm{pH}$ level ranging from 5 to 9 . The most remarkable difference between the tracers is the particle size; however, differences in coating and other constituents are also evident. The coating of the $111 \mathrm{~nm}$ tracer and $59 \mathrm{~nm}$ tracer are both dextran based (dextran and carboxydextran, respectively); however, a polyglucose sorbitol carboxymethylether coating is used in the $32 \mathrm{~nm}$ tracer. Although the coating is known to be a factor influencing in vivo nanoparticle distribution, particle size was demonstrated to be the most significant factor for interstitially administered tracers. ${ }^{19,29,32-34}$ The poor performance of the polyethylene glycol-coated $32 \mathrm{~nm}$ tracer compared to the other tracers is therefore likely explained by a combination of the particle size distribution and the coating. The observed differences between the dextran-coated tracers are most likely explained by the different particle size distributions of these tracers.

\section{Conclusion}

Currently, the $59 \mathrm{~nm}$ tracer is the best performing magnetic nanoparticle tracer, approved for human use, in SLNB with a handheld magnetometer. The particles of the $59 \mathrm{~nm}$ tracer distribute rapidly from the injection site to the SLNs, allowing transcutaneous localization within 30 minutes in contrast to the 240 minutes for the $32 \mathrm{~nm}$ tracer and $111 \mathrm{~nm}$ tracer. The particles of the $59 \mathrm{~nm}$ tracer are retained in the first nodes reached in contrast to the particles of the $32 \mathrm{~nm}$ tracer. Finally, the particles accumulate in high concentrations, facilitating intraoperative localization. The rapid distribution, retention in the first nodes reached, and accumulation in high concentration make the $59 \mathrm{~nm}$ tracer the most suitable magnetic tracer for SLNB.

\section{Acknowledgments}

This research was supported by the Dutch Technology Foundation STW, which is part of the Netherlands Organization for Scientific Research (NWO), which is partly funded by the Ministry of Economic Affairs.

\section{Disclosure}

Although one of the authors (QAP) fulfills a part-time paid advisory role as Chief Scientist (Physics) for Endomagnetics Ltd, his role in this work has been purely academic. The remaining authors report no conflicts of interest and have no financial or personal relationships with other people or organizations that could inappropriately influence their work.

\section{References}

1. WHO. GLOBOCAN 2012 v1.0. Lyon, France: International Agency for Research on Cancer; 2013.

2. Giuliano AE, Kirgan DM, Guenther JM, Morton DL. Lymphatic mapping and sentinel lymphadenectomy for breast cancer. Ann Surg. 1994; 220(3):391-398. [discussion 398-401].

3. Kim T, Giuliano AE, Lyman GH. Lymphatic mapping and sentinel lymph node biopsy in early-stage breast carcinoma: a metaanalysis. Cancer. 2006;106(1):4-16.

4. Krag DN, Weaver DL, Alex JC, Fairbank JT. Surgical resection and radiolocalization of the sentinel lymph node in breast cancer using a gamma probe. Surg Oncol. 1993;2(6):335-339. [discussion 340].

5. Gill G; SNAC Trial Group of the Royal Australasian College of Surgeons (RACS) and NHMRC Clinical Trials Centre. Sentinel-lymph-node-based management or routine axillary clearance? One-year outcomes of sentinel node biopsy versus axillary clearance (SNAC): a randomized controlled surgical trial. Ann Surg Oncol. 2009;16(2):266-275.

6. Krag DN, Anderson SJ, Julian TB; National Surgical Adjuvant Breast and Bowel Project. Technical outcomes of sentinel-lymph-node resection and conventional axillary-lymph-node dissection in patients with clinically node-negative breast cancer: results from the NSABP B-32 randomised phase III trial. Lancet Oncol. 2007;8(10):881-888.

7. Mansel RE, Fallowfield L, Kissin M, et al. Randomized multicenter trial of sentinel node biopsy versus standard axillary treatment in operable breast cancer: the ALMANAC Trial. J Natl Cancer Inst. 2006;98(9):599-609.

8. Veronesi U, Paganelli G, Viale G, et al. A randomized comparison of sentinel-node biopsy with routine axillary dissection in breast cancer. N Engl J Med. 2003;349(6):546-553.

9. Zavagno G, De Salvo GL, Scalco G; GIVOM Trialists. A Randomized clinical trial on sentinel lymph node biopsy versus axillary lymph node dissection in breast cancer: results of the Sentinella/GIVOM trial. Ann Surg. 2008;247(2):207-213. 
10. Rescigno J, Zampell JC, Axelrod D. Patterns of axillary surgical care for breast cancer in the era of sentinel lymph node biopsy. Ann Surg Oncol. 2009;16(3):687-696.

11. Ahmed M, Purushotham AD, Douek M. Novel techniques for sentinel lymph node biopsy in breast cancer: a systematic review. Lancet Oncol. 2014;15(8):e351-e362.

12. Douek M, Klaase J, Monypenny I; SentiMAG Trialists Group. Sentinel node biopsy using a magnetic tracer versus standard technique: the SentiMAG multicentre trial. Ann Surg Oncol. 2014;21(4):1237-1245.

13. Thill M, Kurylcio A, Welter R, et al. The Central-European SentiMag study: sentinel lymph node biopsy with superparamagnetic iron oxide (SPIO) vs radioisotope. Breast. 2014;23(2):175-179.

14. Shiozawa M, Lefor AT, Hozumi Y, et al. Sentinel lymph node biopsy in patients with breast cancer using superparamagnetic iron oxide and a magnetometer. Breast Cancer. 2013;20(3):223-229.

15. Fang C, Zhang M. Multifunctional magnetic nanoparticles for medical imaging applications. J Mater Chem. 2009;19:6258-6266.

16. Wang J, Byrne JD, Napier ME, DeSimone JM. More effective nanomedicines through particle design. Small. 2011;7(14):1919-1931.

17. Jimenez IR, Roca M, Vega E, et al. Particle sizes of colloids to be used in sentinel lymph node radiolocalization. Nucl Med Commun. 2008;29(2): 166-172.

18. O'Brien LM, Duffin R, Millar AM. Preparation of 99mTc-Nanocoll for use in sentinel node localization: validation of a protocol for supplying in unit-dose syringes. Nucl Med Commun. 2006;27(12):999-1003.

19. Swartz MA. The physiology of the lymphatic system. Adv Drug Deliv Rev. 2001;50(1-2):3-20.

20. Anninga B, Ahmed M, Van Hemelrijck M, et al. Magnetic sentinel lymph node biopsy and localization properties of a magnetic tracer in an in vivo porcine model. Breast Cancer Res Treat. 2013;141(1):33-42.

21. McMasters KM, Tuttle TM, Carlson DJ, et al. Sentinel lymph node biopsy for breast cancer: a suitable alternative to routine axillary dissection in multi-institutional practice when optimal technique is used. J Clin Oncol. 2000;18(13):2560-2566.

22. Visscher M, Pouw JJ, van Baarlen J, Klaase JM, Ten Haken B. Quantitative analysis of superparamagnetic contrast agent in sentinel lymph nodes using ex vivo vibrating sample magnetometry. IEEE Trans Biomed Eng. 2013;60(9):2594-2602.

23. Masannat Y, Shenoy H, Speirs V, Hanby A, Horgan K. Properties and characteristics of the dyes injected to assist axillary sentinel node localization in breast surgery. Eur J Surg Oncol. 2006;32(4):381-384.
24. Martin RC 2nd, Edwards MJ, Wong SL, et al. Practical guidelines for optimal gamma probe detection of sentinel lymph nodes in breast cancer: results of a multi-institutional study. For the University of Louisville breast cancer study group. Surgery. 2000;128(2):139-144.

25. Kuehn T, Bauerfeind I, Fehm T, et al. Sentinel-lymph-node biopsy in patients with breast cancer before and after neoadjuvant chemotherapy (SENTINA): a prospective, multicentre cohort study. Lancet Oncol. 2013;14(7):609-618.

26. Visscher M, Waanders S, Krooshoop HJG, ten Haken B. Selective detection of magnetic nanoparticles in biomedical applications using differential magnetometry. J Magn Magn Mater. 2014;365:31-39.

27. Johnson L, Pinder SE, Douek M. Deposition of superparamagnetic iron-oxide nanoparticles in axillary sentinel lymph nodes following subcutaneous injection. Histopathology. 2013;62(3):481-486.

28. Iida S, Imai K, Matsuda S, et al. In vivo identification of sentinel lymph nodes using MRI and size-controlled and monodispersed magnetite nanoparticles. J Magn Reson Imaging. 2013;38(6):1346-1355.

29. Kjellman P, in't Zandt R, Fredriksson S, Strand SE. Optimizing retention of multimodal imaging nanostructures in sentinel lymph nodes by nanoscale size tailoring. Nanomedicine. 2014;10(5):1089-1095.

30. Mori Y, Umeda M, Fukunaga M, Ogasawara K, Yoshioka Y. MR contrast in mouse lymph nodes with subcutaneous administration of iron oxide particles: size dependency. Magn Reson Med Sci. 2011;10(4):219-227.

31. Reimer P, Balzer T. Ferucarbotran (Resovist): a new clinically approved RES-specific contrast agent for contrast-enhanced MRI of the liver: properties, clinical development, and applications. Eur Radiol. 2003;13(6):1266-1276.

32. Hawley AE, Davis SS, Illum L. Targeting of colloids to lymph-nodes influence of lymphatic physiology and colloidal characteristics. Adv Drug Deliv Rev. 1995;17(1):129-148.

33. Oussoren C, Zuidema J, Crommelin DJA, Storm G. Lymphatic uptake and biodistribution of liposomes after subcutaneous injection.2. Influence of liposomal size, lipid composition and lipid dose. Biochim Biophys Acta-Biomemb. 1997;1328(2):261-272.

34. Maiseyeu A, Bagalkot V. In vitro uptake of apoptotic body mimicking phosphatidylserine-quantum dot micelles by monocytic cell line. Nanoscale Res Lett. 2014;9(1):176.
International Journal of Nanomedicine

\section{Publish your work in this journal}

The International Journal of Nanomedicine is an international, peerreviewed journal focusing on the application of nanotechnology in diagnostics, therapeutics, and drug delivery systems throughout the biomedical field. This journal is indexed on PubMed Central, MedLine, CAS, SciSearch $®$, Current Contents $\AA /$ Clinical Medicine,

\section{Dovepress}

Journal Citation Reports/Science Edition, EMBase, Scopus and the Elsevier Bibliographic databases. The manuscript management system is completely online and includes a very quick and fair peer-review system, which is all easy to use. Visit http://www.dovepress.com/ testimonials.php to read real quotes from published authors. 